\title{
Calculated hierarchy model of the process of metals dynamic failure
}

\author{
A.Ya. Uchaeva , S.S. Sokolov, N.I. Selchenkova, E.V. Kosheleva, and L.V. Zhabyka \\ Russian Federal Nuclear Center - VNIIEF, Nizhni Novgorod Region, Sarov 607188, Russia
}

\begin{abstract}
In the paper there are considered dynamic properties of dissipative structures that make it possible to get time dependence of cascade accumulation of failure centers at different stages of failure process. There is presented a hierarchy model of dynamic failure based on wide experimental materials, mathematical formalism of percolation theory, fractal geometry.
\end{abstract}

\section{Introduction}

The investigation of micro-failures occurrence in the phenomenon of dynamic failure under the effect of shortpulse loads of high intensity is highly acute today. As a rule high-intensity pulse effect leads to the phenomenon of dynamic failure as a result of which there appear dissipative structures (DS). It has been demonstrated before that resistance against external effect is offered by DS arising in a failed body, for example, cascade of failure centers, which quantitative characteristics define the statistical picture of the process of dynamic failure. The investigation of metals behavior under extreme conditions is possible by example of studying the process of dynamic failure taking place under the effect of high-power pulses of penetrating radiation $[1-4]$.

\section{Results and discussion}

It is well-known that time dependences of the process of metals dynamic failure are conditioned by hierarchic properties of DS - cascade of failure centers defining the process of metals dynamic failure within the $t \sim 10^{-6} \div$ $10^{-10} \mathrm{~s}$. The formation of DS is possible only at joint realization of several structural levels while the fractal dimensionality is a quantitative characteristic [2,3]. The process of failure centers accumulation in different metals under the phenomenon of dynamic failure is defined by ratio [1-4]:

$$
\langle D\rangle / r=\langle D\rangle / N^{-1 / 3}=1 / 2.7,
$$

where $N$ - number of failure centers, $r$ - average distance between failure centers, $\langle D\rangle$ - average size of failure centers. When failure centers, cracks start enlarging and when concentration of failure centers for example, reaches the value of $\sim 5 \%$, there starts forming the other level of DS. Fig. 1 gives the results of processing microsections of copper, bronze, iron samples after the effect of relativistic electron beams (REB) [3] and samples of copper under the effect of tensile stresses [5]. The data given in Fig. 1 demonstrate concentration criterion for DS at different scale-time levels.

The cascade of failure centers (meso-level 1) and roughness of failure surface (macro-level) on the threshold

a e-mail: uchaev@expd.vniief.ru of macro-failure when there takes place a topological phase transition - occurrence of connectivity in the system of failure centers - are percolation clusters. The universal behavior of metals in the phenomenon of dynamic failure defines the possibility of applying the apparatus of the theory of critical phenomena [6] to the qualitative description of the process and makes it possible to forecast the behavior of uninvestigated materials within the $t \sim 10^{-6} \div$ $10^{-10} \mathrm{~s}$ longevity range; the range of initial temperatures is $T_{0} \sim 4 K-0.8 T_{m}[1,2]$. The analysis of current literary data shows that today there are no physical models that use the concept of structural levels hierarchy, methods of nonlinear physics, mathematical apparatus of the theory of critical phenomena, theory of percolation for the solution of the task of describing dynamic failure of metals, for example in the mode of pulsed volumetric heating [4]. Earlier there has been found the invariant connecting parameters of internal effect - pressure $\mathrm{P}(\mathrm{t})$ with energy parameters of crystalline lattice: $H$ - enthalpy and $L_{m}$ - latent heat of fusion. Dynamic invariant $I(P, t)=P(t) / \Gamma \rho\left(H+L_{m}\right)$ has close values for a set of investigated metals within the $t \sim 10^{-6} \div 10^{-10}$ s longevity range ( $\Gamma-$ Gruneisen parameter, $\rho$ - metal density) [1-3]. This testifies to the fact that for a definite value of critical pressure $P(t)$ for each metal at a definite value of longevity $t$, the time dependences of the number of failure centers $N(P, t)$ and the rate of centers formation $J(P, t)$, characterizing kinetics of failure process, have close values. The spectral distribution of failure centers by sizes has the following form:

$$
N_{i}\left(D_{i}\right) \sim D_{i}^{-\alpha},
$$

where $N_{i}$ - number of failure centers of $D_{i}$ size, $1<\alpha<2$, $\alpha-$ fractal dimensionality [1-3]. The cascade of failure centers is a self-similar cluster and dimensions $D$ of failure centers form geometric progression

$$
D_{i}=q D_{i-1}=q^{i} L
$$

where $L-$ size of the area (volume) occupied by failure centers; parameter $\mathrm{q}<1$.

The data of microsections fractografic researches for a set of metals demonstrate that the functions of the rate of centers formation $J(t)$ in coordinates $t / t_{r}, J / J_{r}\left(t_{r}-\right.$ failure duration, $t$-current time, $J_{r}$ - maximal value of the rate of centers formation) for different metals within the $t \sim 10^{-6} \div 10^{-10}$ s longevity range. 


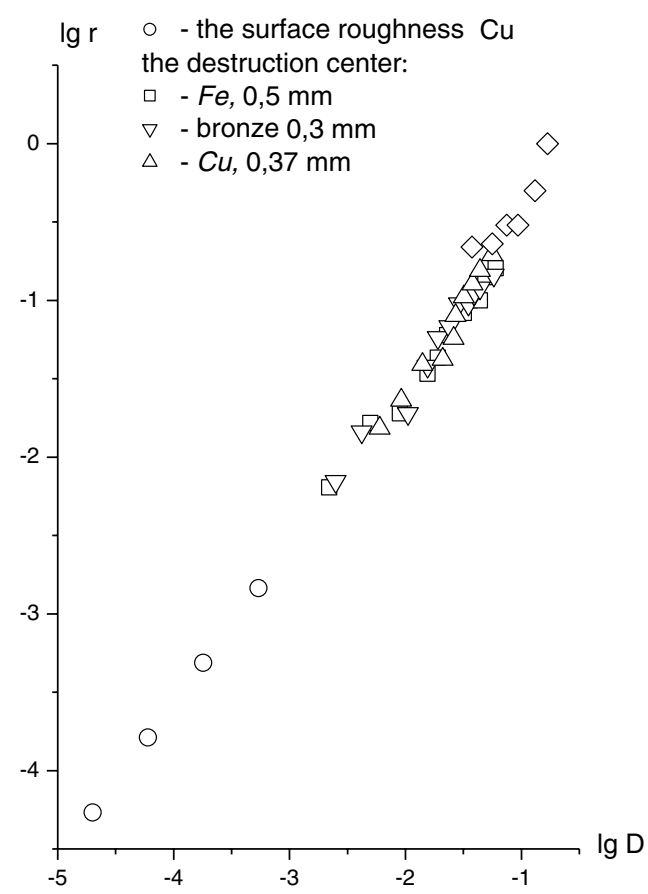

Fig. 1. Concentration criterion ([r], [D] - in $\mathrm{mm})$.

Fig. 2 gives the data on the rate of centers formation $J(t)$ for various metals. The rate of centers formation $J(t)$ has the following form

$$
J(t) \approx A \cdot \exp (t / B)^{\lambda} \cdot(1-t)^{\beta},
$$

where $\lambda, \beta$-degree indexes, $A, B$ - constants.

If $\lambda<1, \beta<0$ expression (4) describes the initial stage of the process of centers formation, when the density of failure centers is low and their interaction can be neglected. If $\lambda<1, \beta>1$ expression (4) describes the final stage of the process of centers formation when there appears interaction between failure centers and their cascade grows as a self-similar object. In this case there arises the dependence of the average distance between failure centers on their size $D$ (see Fig. 1) $[3,4]$. The rate of centers formation expressed through an invariant is

$$
J(t)=J_{t o t}\left(t_{r}\right) \exp \left(t I\left(t_{r}\right) / A\right)^{\lambda}\left(1-\left(t I\left(t_{r}\right) / A\right)^{\beta}\right),
$$

where $A$ - const. The ratio for the rate of centers formation makes it possible to include kinetics of the process of dynamic failure to the hierarchic model that allows adequate description of metals behavior under extreme conditions.

\section{Conclusions}

There is presented the hierarchic model of dynamic failure based on extensive experimental material, mathematical formalism of percolation theory, fractal geometry. Within the framework of the model it is demonstrated that nonequilibrium system - loaded body - progresses through

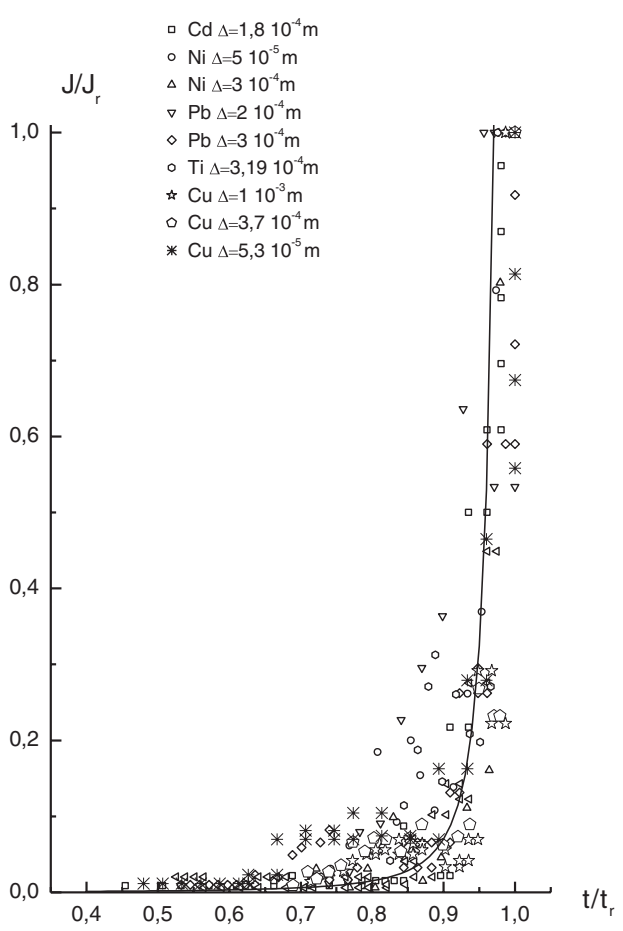

Fig. 2. Rate of centers formation in $t / t_{r}, J / J_{r}$ coordinates $[3,4]$.

transition from one scale-time level to another while the concentration criterion construes as one of basic parameters characterizing non-equilibrium dynamic process on various scale-time levels. In the advanced hierarchic failure model there is established the time dependence of critical pressure leading to failure what made it possible to express in a definite mathematical form a set of kinetic processes that were introduced to multidimensional mathematical codes.

\section{References}

1. R.I. Il'kaev, A.Ya. Uchaev, S.A. Novikov, N.I. Zavada, L.A. Platonova, N.I. Sel'chenkova. DAN 384, 3 (2002)

2. R.I. Il'kaev, V.T. Punin, A.Ya. Uchaev, S.A. Novikov, N.A. Yukina, DAN 393, 3 (2003)

3. E.K. Bonyushkin, N.I. Zavada, S.A. Novikov, A.Ya. Uchaev. Kinetics of dynamic metals failure under the mode of pulse volume heat-up (Sarov, RFNC-VNIIEF Press, 1998)

4. R.I. Il'kaev, V.T. Punin, A.Ya. Uchaev, N.I. Sel'chenkova, L.A. Platonova, Ye.V. Kosheleva. Ye.A. Veselova, L.V. Zhabyka. Nuclear physics and engineering 1, 1 (2010)

5. Kh.G. Killian, V.I. Vettegren, V.N. Svetlov. Solid state physics 43, 11 (2001)

6. A.Z. Patashinski, V.L. Pokrovski, Fluctuation theory of phase transitions. (Moscow: Nauka, 1982) 